\title{
Zur Kenntniss der Tubengonorrhoe.
}

\author{
Von \\ Dr. Adolf Schmitt \\ in Würzburg.
}

Es ist Nöggerath's unbestrittenes Verdienst, durch seine 1872 erschienene Schrift ${ }^{1}$ ) auf die hohe Wichtigkeit der Gonorrhoe beim Weibe zuerst eindringlich aufmerksam gemacht zu haben. Man hatte bis dahin kaum eine Ahnung, viel weniger eine genauere Kenntniss davon, dass so ausserordentlich häufig die Gonorrhoe sich vom Uterus aus auf dessen Anhänge weiterverbreitet und dass die Gonorrhoe eine so hervorragende Bedeutung in der Aetiologie so vieler Frauenkrankheiten besitzt.

Zwar wurden Nöggerath's Anschaungen und Behauptungen über die von ihm aufgestellte, ,latente" Gonorrhoe von Vielen bestritten, aber das, was er über die Bedeutung der acuten und noch viel mehr der chronischen Gonorrhoe für das Entstehen der Tubenerkrankungen und ihrer Folgen, der Entzündung des Beckenperitoneum sagt, ist von vielen Forschern angenommen und bestätigt worden.

Bekanntlich unterscheidet Nöggerath drei Formen in der Entzïndung des Beckenperitoneum: 1) die acute Perimetritis (mit und ohne Parametritis); 2) die recurrirende Perimetritis im Zusammenhange mit gonorrhoischer Salpingitis und 3) die chronische Perimetritis.

Als Ausgangspunkt und Ursache dieser drei Formen von Perimetritis, vor allem der recurrirenden, nimmt er die Gonorrhoe an, die sich auf die Tuben fort- und in denselben festgesetzt hat, um hier als dauernder Herd für neue Exacerbationen der Entzündung meist für Lebenszeit zu bestehen; die Salpingitis go-

1) Die latente Gonorrhoe beim Weibe. Bonn 1872. 
norrhoica ist also nach seiner Ansicht eine ganz ausserordentlich häufige, ja nahezu beständige Complication der Gonorrhoe.

Dass Nöggerath's Ansichten, die so neu und überraschend kamen, auf manchen starken Zweifel und Widerspruch gestossen sind, ist wohl begreiflich, und doch wurden eigentlich stichhaltige Gründe zu seiner Widerlegung nicht vorgebracht; es hat sich vielmehr, wie Schroeder') sagt, gerade in neuester Zeit die Mehrzahl der Gynäkologen davon überzeugt, dass Nöggerath's Ansichten im wesentlichen richtig sind. Schroeder selbst weicht nur darin von ihm ab, dass er glaubt, der Tripper des Mannes könne wohl, müsse aber nicht immer die von Nöggerath angenommenen üblen Folgen haben. Auch nach Fritsch ${ }^{2}$ ) kann Perimetritis im Anschlusse an Gonorrhoe auf dreifache Weise entstehen: Es kann sich 1) der Lymphraum der Peritonealhöhle entzünden, oder 2) die Entzündung durch die dünne Tubenwand hindurch auf den peritonealen Ueberzug fortsetzen, oder 3) es gelangt der virulente Eiter der Tubengonorrhoe durch Ueberfliessen in die Peritonealhöhle und ruft hier durch Ueberimpfung eine Perimetritis hervor.

Ich werde auf diese Anschauungen wieder zurückkommen, erwähne aber gleich hier schon, dass von einer, gewissermaassen durch Impfung entstandenen, auf unmittelbarer Gonococcenwirkung beruhenden Perimetritis nach den neueren Beobachtungen keine Rede sein kann.

L. Tait ${ }^{3}$ ) und Sänger ${ }^{4}$ ) schlossen sich den Ansichten Nöggerath's an.

Nach Sänger's Berechnung — die sich allerdings nur auf Anamnese und klinischen Befund, nicht auch auf mikroskopische Untersuchung stütat - ist die Gonorrhoe in $1 / 9$ aller gynäkologischen Erkrankungen als Ursache anzusehen und von allen jemals gonorrhoisch inficirten Frauen hat die Hälfte an den Folgen der Infection, an Erkrankung der Uterusanhänge zu leiden. Als

1) Krankheiten der weiblichen Geschlechtsorgane. 1886.

2) Die Krankheiten der Frauen. 1886.

3) Pyosalpinx following gonorrhoea. New-York med. Journal XL. 1884.

4) Ueber gonorrhoische Erkrankungen der Uterusadnexe u. s. w. Dieses Archiv 1885, Bd. XXV, S. 126. - Die Beziehungen der gonorrhoischen Erkrankungen zu Puerperalerkrankungen. Gynäkologen-Congress 1886. - Aetiology, Pathology and Classification of Salpingitis. Brief an 1D. T. Nelson als Antwort für L. Tait. 1886. 
der Hauptherd aber, welcher dauernd besteht, von wo aus immer wieder Nachschübe und Entladungen in die Bauchhöhle erfolgen, sind die Tuben zu betrachten.

Auf dem gynäkologischen Congresse 1886 hielt Säng er seine frühere Ansicht über die Möglichkeit einer Parametritis gonorrhoica nicht mehr aufrecht, indem er die Gonorrhoe ausdrücklich als eine Oberflächenerkrankung des Genitalschlauches und des Beckenbauchfelles bezeichnete - eine Ansicht, welche durch die auf der von Rinecker'schen Klinik zu Würzburg erwiesene und durch $\mathrm{Bumm}$ völlig sicher gestellte Thatsache bestätigt wird, dass der Gonococcus nur in die allerobersten submucösen Gewebe einzudringen vermag und in Bindegewebe sehr rasch zu Grunde geht. Sänger nimmt also eine gleichzeitig mit Perimetritis bestehende Parametritis als durch Mischinfection entstanden an, wobei aber auch die Perimetritis nicht als durch unmittelbare Wirkung der Gonococcen hervorgerufen zu denken ist.

Die gonorrhoische Infection in und ausser dem Wochenbette hält Sänger im Gegensatze zu den eigentlichen schweren Puerperalinfectionen nicht für eine unmittelbar lebensgefährliche Erkrankung, vor allem deshalb nicht, weil allgemeine Peritonitis niemals an gonorrhoische Perimetritis sich anschliesse. Kommt dennoch kurze Zeit nach dem Austritte von Tubeneiter in die Bauchhöhle ein Todesfall vor, so nimmt er Shok, vor allem aber Mischinfection mit den Mikroorganismen der verschiedenen Formen von acuter Wundinfection (Septikämie, Pyämie u. s. w.) als Ursache an. Doch ist eine grosse Anzahl der mit "Puerperalfieber" bezeichneten Erkrankungen, besonders wenn sie ganz acut, stürmisch und schwer im Beginne einsetzen, dann aber verhältnissmässig leicht, aber ungemein langsam verlaufen, auf Rechnung gonorrhoischer Infection zu setzen, und zwar ist es theils Spätinfection im Wochenbette (zweite bis dritte Woche nach der Geburt), theils ältere, schon vor der Schwangerschaft bestandene Gonorrhoe, welche die Ausbildung von Tubenerkrankungen verursacht.

Den schwersten Einwand gegen Sänger's Aufstellungen erhob Kroner ${ }^{1}$ ) mit der Forderung, dass der sichere Beweis für den gonorrhoischen Ursprung der Erkrankung der Uterusanhänge durch das Auffinden der Gonococcen geliefert werden müsse, ein Punkt, auf den von Sänger allerdings gar kein Gewicht gelegt worden ist.

1) Naturforscher-Versammlang zu Magdeburg 1884. 
In der Versammlung der deutschen Naturforscher zu Berlin 1886 berichtete Gusserow ${ }^{1}$ ) über 14 Fälle von Tubenexstirpation. Die in allen diesen Fällen bestehende Perimetritis betrachtet Gusserow (ätiologisch) als das Wesentliche und führt sie zurück - in den allermeisten Fällen wenigstens - auf gonorrhoische Infection, welche zu eitrigem Tubenkatarrh und dann zur Entzündung der Serosa der Uterusanhänge geführt habe. Auf derselben Versammlung gab Martin, gestützt auf 287 von ihm beobachtete Fälle von Tubenerkrankung, an, dass die Hälfte dieser Fälle als Erzeugniss einer fortschreitenden katarrhalischen Erkrankung der Uterusschleimhaut zu betrachten sei; die übrigen Fälle gehören wieder zur einen Hälfte der puerperalen, zur anderen der gonorrhoischen bezw. tuberculösen Erkrankung an in einem Verhältnisse von $6: 1$.

Bei der grossen Zahl der zur Beobachtung gelangten Tubenerkrankungen darf es nicht wundern, dass bei Gelegenheit von Salpingotomien und Autopsien von vielen Seiten Untersuchungen auf Gonococcen im Tubensecrete und in den perimetritischen Exsudaten angestellt wurden, und es ist auffallend, dass so wenig bejahende Ergebnisse erzielt wurden. Westermark ${ }^{2}$ ) und $0_{r t h-}$ mann ${ }^{3}$, auf deren Befunde ich'später noch einzugehen habe, waren bisher die Einzigen, welche Gonococcen im Tubeneiter sicher nachweisen konnten, während der Fall von $\mathrm{Ceppi}^{4}$ ), der in einem von der Vagina aus geöffneten Exsudate Gonococcen gefunden hat, ganz vereinzelt dasteht; dagegen ist es bis heute noch niemals gelungen, in Schnittpräparaten in der Tubenschleimhaut, bezw. ihrem Epithel, Gonococcen zu sehen.

Der Verlauf der gonorrhoischen Infection auf der inficirten Schleimhaut und die durch dieselbe gesetzten Veränderungen sind nach den Untersuchungen Bumm's ${ }^{5}$, Bockhart's ${ }^{6}$ ), Stein-

1) Dieses Archiv 1887.

2) Ein Fall von Salpingitis gonorrhoica mit Gonococcen im Exsudate. Hygiea 1886.

3) Virchow's Archiv 1887.

4) Revue médicale de la Suisse 1887.

5) Beitrag zur Kenntniss der Gonorrhoe der weiblichen Genitalien. Dieses Archiv 1884. - Der Mikroorganismus der gonorrhoischen Schleimhauterkrankungen "Gonococcus Neisser". Wiesbaden 1888. - Ueber gonorrhoische Mischinfection beim Weibe. 60. deutsche Naturforscher-Versammlung 1887.

6) Beitrag zur Aetiologie und Pathologie des Harnröhren-Trippers. Verhandlungen der Würzburger physikalisch-medicinischen Gesellschaft 1885. 
schneider's ${ }^{1}$ ) u. A. genügend bekannt und studirt. Aus allen Untersuchungen aber geht hervor, dass an das Vorhandensein von Cylinderepithel die Möglichkeit der Gonococceneinwanderung gebunden zu sein scheint, denn fast alle Beobachtungen haben gleichmässig ergeben, dass die Pilzeinwanderung überall da scharf abschneidet, wo der Uebergang von Cylinderepithel in Plattenepithel stattfindet, dass also die Coccen nicht im Stande sind, das Hinderniss einer zusammenhängenden Lage unversehrten, verhornten Plattenepithels zu überwinden, dass vielmehr alle der gonorrhoischen Infection zugänglichen Schleimbäute von Cylinderepithel überkleidet sind oder doch eine Epitheldecke haben, die dem Cylinderepithel nahe steht. Das Epithellager ist dabei nur von geringer Dicke, das Protoplasma der zelligen Elemente recht zart.

Was die Art und Weise der Verbreitung der Infection über eine Schleimhautfläche betrifft, so ist eigentlich nur bei einer Stelle eine gleichzeitige und gleichmässige Vertheilung des contagiösen Stoffes über die ganze Ausdehnung der Schleimhaut anzunehmen: bei der Conjunctiva. Durch den ständigen und. gleichmässigen Lidschlag sind auch die Bedingungen zu einer gleichzeitigen Vertheilung über den ganzen Conjunctivalsack gegeben, während bei allen übrigen Schleimhäuten die contagiöse Entzündung von dem zuerst ergriffenen Orte aus nur allmälig fortschreitet, so dass in derselben Strecke an der einen Stelle lebhafteste Entzündung und Absonderung mit massenhaften Gonococcen sich finden kann, während andere Stellen schon im Stadium der Wiederherstellung begriffen und ganz oder theilweise mit neugebildetem Epithel überzogen sind und keine Spur von Gonococcen mehr zeigen, wieder andere aber überhaupt noch nicht ergriffen und völlig gesund sind. Andererseits aber kann an Stellen, welche schon in Heilung begriffen sind, eine neue Cocceneinwanderung, ein Rïickfall stattfinden, wenn nämlich das neu gebildete Epithellager dem allzuheftigen Andrange der auswandernden Rundzellen - die ja im allgemeinen die Epitholneubildung nicht verhindern - nicht zu widerstehen vermag, sondern ähnlich wie bei der ersten Infection auseinandergerissen und abgehoben wird.

1) Ueber den Sitz der gonorrhoischen Infection beim Weibe. Berliner klinische Wochenschrift 1887. 
Wie es scheint, treten aber der Weiterverbreitung des gonorrhoischen Vorganges auf die ganze Ausdehnung eines zusammenhängenden Schleimhautschlauches auf ihrem Wege $a b$ und zu Hindernisse entgegen in Gestalt der zwischenliegenden, anatomisch engen Stellen, z. B. des Orificium internum uteri. und der uterinen Tubenmündungen; es müssten sonst, bei dem so ähnlichen Baue der Schleimhäute, der Uterus und die Tuben noch viel öfter in den Vorgang mit einbezogen werden, als dies - soweit wenigstens bis jetzt unsere Kenntniss reicht - der Fall zu sein scheint. Sobald allerdings günstige Vorbedingungen (Reize aller Art, Ausschweifungen in venere et baccho, mechanische Einwirkungen) die Ueberwindung dieser Hindernisse ermöglichen, schreitet der Vorgang jenseits derselben in der gleichen Art fort, wie auf der ursprünglich ergriffenen Schleimhant. Eine Verschiedenheit zwischen den früheren Ansichten und den neueren über die Art der Weiterverbreitung liegt jedenfalls darin, dass man früher, als die Gonorrhoe für eine katarrhalische Erkrankung galt, ein einfaches Weiterschreiten auf der einmal ergriffenen Schleimhaut annahm, während jetzt nur das Vordringen der specifischen Mikroorganismen als Ursache der Ausdehnung und Verbreitung der gonorrhoischen Entzïndung über einen Schleimhauttractus angesehen werden muss.

Wenn man auch annehmen darf, dass die Gonorrhoe auf der Tubenschleimhaut ganz ähnliche Veränderungen hervorruft, wie auf anderen Schleimhäuten von gleicher oder ähnlicher anatomischer Beschaffenheit, so ist es natürlich doch von Bedeutung, diese Veränderungen an der erkrankten Tube selbst festzustellen. Trotzdem jetzt - infolge der grossen Zahl von Operationen viel Material gewonnen wird, so sind doch nur wenige derartige Untersuchungen veröffentlicht worden.

Mit der Untersuchung der Entzündung an den Tuben und der durch dieselbe gesetzten Veränderungen hat sich in jüngster Zeit Orthmann ${ }^{1}$ ) eingehend beschäftigt. Bei Salpingitis purulenta fand er das Oberflächenepithel fast vollkommen geschwunden, nur in den Tiefen der Schleimhautfalten und als Auskleidung der Hohlräume kommt es noch erhalten vor. Eitrige Schmelzung und umgrenzte Nekrose führen zu einer nahezu vollständigen Zerstörung der Schleimhaut, die bis auf die Muskelschicht hinab-

1) a. a. 0 . 
reichen kann. Die Tubenwand ist erheblich verdickt, vor allem das intermuskuläre Bindegewebe, während die Muskulatur unverändert oder verkümmert erscheint. Als Ursache der Salpingitis purulenta nimmt Orthmann das Puerperium und hauptsächlich die Gonorrhoe an, ebenso wie für Pyosalpinx, bei Verschluss des Ostium abdominale oder bei Verlöthungen der Tubenwandungen an einer Stelle unter sich. Auch hier zeigt sich die Schleimhaut nahezu vollkommen zerstört, die Wandungen dagegen infolge ziemlich gleichmässiger Hypertrophie des Muskel- und Bindegewebes ziemlich stark verdickt und stark infiltrirt. Es sind diese kurzen Angaben gewissermaassen das Mittel aus einer grösseren Anzahl von 0 rthmann untersuchter Präparate. In den meisten der diesen Untersuchungen zu Grunde liegenden Fälle wurden von Orthman $\mathrm{n}$ weder in der Absonderung, noch in zahlreichen Schnitten Mikroorganismen gefunden.

Westermark ${ }^{1}$ ) war der Erste, der in dem eitrigen Inhalte einer exstirpirten Pyosalpinx mit aller Sicherheit Gonococcushaufen nachgewiesen hat. Ferner berichtete 0 rthmann ${ }^{2}$ ) über einen zweiten Fall, wo die Ovario-Salpingotomia duplex an einer 22jährigen Kranken, deren Mann wiederholt gonorrhoisch inficirt war, vorgenommen werden musste, und sich in den sofort nach der Operation angefertigten Trockenpräparaten von Eiter der rechten Tube - bei einer Doppelfärbung von Fuchsin-Methylenblau - in jedem Gesichtsfelde zwei und mehr Eiterzellen fanden, welche an der charakteristischen Form und Grösse deutlich zu erkennende Gonococcenhaufen enthielten. GentianaviolettFärbung zeigte dasselbe Ergebniss, während die Gram'sche Färbungsmethode verneinend ausfiel. Andere Mikroorganismen waren weder in den Präparaten, noch durch Impfung nachweisbar; ebenso hatte eine genaue Durchmusterung zahlreicher, auf verschiedene Art gefärbter Schnittpräparate ein verneinendes Ergebniss. Das Oberflächenepith el der Schleimhaut war vollkommen geschwunden, die Schleimhautfalten zu mehr weniger hohen Wülsten verschmolzen, die tieferen Ausbuchtungen in abgeschlossene, mit Cylinderepithel ausgekleidete Hohlräume verwandelt; die Schleimhaut selbst ist hochgradig infiltrirt, zeigt an manchen Stellen deutliche eitrige Schmelzung. Gefässe sind nur in ge-

1) a. a. 0

2) Berliner klinische Wochenschrift 1887, Nr. 14. 
ringer Zahl vorhanden; die übrige Tubenwand ist ebenfalls stark infiltrirt, die Muskulatur verdünnt, das intermuskuläre Bindegewebe stark vermehrt. Die Gefässe sind hier zahlreich und dickwandig.

Ich konnte diesen Befund an einem Stücke derselben Tube, das Dr. Orthmann an Dr. Bumm schickte und das ich in zahlreichen Präparaten auf Gonococcen im Gewebe untersuchte, vollkommen bestätigen.

Aus der Vorgeschichte des Falles, dem die beiden von mir untersuchten Tuben entstammen, ist Folgendes anzuführen:

Die Kranke ist $26 \frac{1}{2}$ Jahre alt, ledig, erblich durchaus nicht. belastet. Menses waren stets regelmässig. Sie hat Anfangs 1886 einmal geboren. Nach ihrer Entbindung hat sie oft geschlechtlich verkehrt, angeblich mit demselben Manne, und Anfangs December zum letzten Male. Nach etwa drei Wochen begann eitriger Ausfluss aus den Genitalien, der später sehr reichlich wurde; die anfänglich ganz unbedeutenden Schmerzen wurden später, besonders im Unterleibe, ausserordentlich heftig.

Befund im Anfange des März: Scheideneingang blassroth gefärbt, zeigt stark entwickelte Carunkeln. Aus der Urethra entleert sich auf Druck etwas milchig aussehender Eiter. Die Cervix steht sehr tief, ist lang, verdickt, hat seitliche Einrisse; Orificium externum geöffnet. Aus der Cervix entleert sich glasiger Schleim. Hintere Muttermundslippe etwas geschwürig. Inguinal-, Cubital- und Cervicaldrüsen sind deutlich geschwollen, nicht empfindlich. Im Rachen nichts Abnormes, kein Leukoderma colli. Im Urethral- und Cervicalsecrete finden sich zahlreiche Gonococcen.

Nach vierwöchentlicher Behandlung mit Tannin-Glycerin verschwinden die Gonococeen im Schleime, während der Ausfluss fortdauert. 26. April trat unter starkem Fieber sehr heftige Schmerzhaftigkeit im Bauche auf, die auf ,parametrische Entzündung" zurückgeführt wurde. Eisblase und Opium. 28. April: Temperatur 40,20, durch Antifebrin auf die Norm herabgedrückt, Bei sehr schlechtem Allgemeinbefinden dauert die Schmerzhaftigkeit im Bauche in wechselnder Stärke, meist sehr heftig, fort. Heisse Breiumschläge. Fieberlose Zeiten von 2-8-14 Tagen Dauer wechseln mit gleich langen mit erhöhter Temperatur $\left(38,0-39,5^{\circ}\right)$ ab. Der Bauch erscheint in seinen unteren Theilen resistent, hochgradig druckempfindlich. Die linke Tube lässt sich als wurstförmiger, ziemlich harter und dicker Strang durchfühlen und scheint an die seitliche Beckenwand durch fächerförmige Verwachsungen befestigt. Diagnose: Pyosalpinx sinistra.

Operation, ausgeführt von Professor Dr. Nieberding, am 20. Juli. Salpingotomia et Ovariotomia sinistra.

Es finden sich sehr ausgedehnte Verwachsungen zwischen Darm, Tube and Ovarium. Bei Lösung derselben tritt Eiter durch das 
Ostium abdominale tabae in die Bauchböhle aus. Darmserosa (am Dickdarme) an einer Stelle eingerissen. Linkes Ovarium ist wallnussgross, cystös entartet, wird trotz starker Verwachsungen, welche die Lösung sehr schwierig machen, mitentfernt. Rechte Tube und rechtes Ovarium lassen keine wesentliehen Veränderungen erkennen und werden deshalb belassen. - Dauer der Operation $1 \frac{1}{2}$ Stunden.

In den ersten 24 Stunden nach der Operation ist das Allgemeinbefinden verhältnissmässig gut, ohne Fieber. Am zweiten Tage 'Temperatur 38,5-39,40. Der Puls, bisher 76-80, wird sehr klein, steigt auf 120-180 Schläge. Verfall der Gesichtszïge; unerträglicher Meteorismus, Tod.

Section am 25. Juli: Der ganze Darm stark durch Gas aufgetrieben. Die Dünndarmschlingen geröthet, an Anlagerungsstellen blasser. Im oberen Theile der Bauchhöhle eine wenig getrübte Flüssigkkeit. Mesenterium serös-blutig infiltrirt; Netz, zum grössten Theile frei, an seinem rechten Rande mit einer Gruppe Dünndarmschlingen verwachsen. (Frische Peritonitis.) In der linken Inguinalgegend am $S$ romanum und am Anfange des Rectum eine umfängliche, stark geröthete, an anderen Stellen mehr gelbliche Anschwellung. Am Eingange ins grosse Becken findet sich eine gelbbraune, anscheinend fäeale Flüssigkeit. Nach Hinwegnahme des grössten Theiles des Darmes erscheint die Operationswunde in gutem Granulationszustande. Das Peritoneum stark geröthet, mit Blutpunkten durchsetzt. S romanum mit dem Peritoneum fest verwachsen. Bei Druck auf das $\mathrm{S}$ romanum vermehrt sich die Flüssigkeit im Becken, doch lässt sich auch bei kräftiger Einspritzung ins Reetum und $\mathrm{S}$ romanum keine Durchbruchsöffnung nachweisen. - Die rechte Tube katarrhalisch entzündet, verdickt, vergrössert, ihre Bauchöffnung durch Bindegewebe überwachsen. Aus der geöffneten Tube entleert sich graugelber Eiter; nach Abspülen des Eiters zeigt sich die Schleimhaut geschwellt und in viele Längsfalten erhoben. Gegenüber der Operationsstelle ist das Rectum von der Serosa entblösst, unterhalb dieser Stelle mit dem sehr kleinen Reste des linken Ovarium, der sich hier befindet, auf das innigste verwachsen. Corpus uteri verhältnissmässig gross: zwischen den Tubenmündungen $6,5 \mathrm{~cm}$ breit, Körperhöhe $4 \mathrm{~cm}$. Die Schleimhaut des Uterus hochroth, die Gefässe an der Oberfläche stark ausgedehnt; starke Eiterbildung. Die Muskulatur durehweg weiss und schlaff. Die Schleimhaut der Harnblase normal; aus der Urethra entleert sich eitrige Flüssigkeit. Bei genauerem Suchen findet sich $10 \mathrm{~cm}$ unterhalb der Valvula Bauhini eine erbsengrosse Durchbruchsöffoung mit stark infltrirter, gerötheter Umgebung.

In dem Eiter der beiden Tuben wurden mit voll. kommener Sicherheit Gonococeen gefunden, und zwar auf folgendem Wege: Aus der bei der Operation entfernten Tube wurde sofort der Eiter in der Weise entnommen, dass mit sterilisirtem Messer senkrecht zur Längsachse der Tube Durchschnitte angelegt wurden; der aus der Höhlung hervorquellende Eiter wurde dann auf Objectträger ausgestrichen. 
Rei der Färbung mit wässeriger Lösung von Fuchsin und Methylenviolett fand sich: 1) als Hauptbestandtheil des Eiters weisse Blutzellen, meist stark körnig; die Kerne nur selten mehr deutlich ausgeprägt, viele Zellen bereits ganz körnig zerfallerı. ') 2) Spärlich, in jedem zweiten oder dritten Gesichtsfelde ein oder zwei Häufehen und vereinzelte Exemplare von Gonococen. Das Aussehen dex Häufchen wie der Einzelexemplare zeigte alle bestimmten Merkmale der Gonococcen, so dass über diesen Punkt, bei genanester Durchsicht der Präparate, kein Zweifel herrschen konnte. Bei der Färbung nach der Gram'schen Methode gaben die Pilze ihren Farbstoff ab, wie dies ja für die Gonococcen gegenüber anderen ähnlichen Mikrobien charakteristisch ist.

Als beim Durchschnitte der an der Leiche noch vorhandenen Tube ebenfalls spärlicher Eiter hervorquoll, wurde derselbe ebenfalls untersucht und lieferte bei gleicher Methode dasselbe Ergebniss. Uterus- und Cervixabsonderung enthielten bei der schon ziemlich weit vorgeschrittenen Verwesung massenhaft Stäbchen und Coccen aller Art, Gonococcen aber konnten nicht mit Bestimmtheit darin nachgewiesen werden.

Die Tuben, welche mir Herr Dr. Bumm in liebenswürdigster Weise zur Untersuchung überliess, wurden sofort nach der Operation bezw. Section in passende Stücke geschnitten und dieselben in absoluten Alkohol eingelegt, später in Paraffin eingeschmolzen und in Schnitte zerlegt. Ueber die angewandto Färbung der Schnitte zum Zwecke der Untersuchung auf Gonococcen -- worauf es ja in erster Linie abgesehen war -, sowie die Feststellung der histologischen Veränderungen werde ich später berichten.

Es ergab sich aus der Untersuchung zahlreicher Präparate folgender a natomischer Befund an den einzelnen Tubenstücken:

1. Stück aus der bei der Operation exstirpirten linken Tube, nahe dem aterinen Ende derselben: Die Schleimhautfalten sind im allgemeinen niedrig, ihre Verästelungen schwach ausgebildet. Man sieht meist nur papillomatöse Erhebungen mit wenig Verzweigungen in die Höhlung der Tube hervorragen. Nur vereinzelte Falten sind höher und ragen mit mehreren Verzweigungen bis etwa in die Mitte der Tubenhöhlung. Während sich zwischen den Lamellen der Ringmuskulatur, die gegen die Norm etwas verdickt erscheint,

1) In auffallendem Gegensatze zu diesem Befunde steht eine Nachricht von Cornil und Terillon (Archives de Pbysiologie normale et pathologique 1887), welche den Inhalt einer gonorrhoischen Tube fast vollständig aus den abgestossenen Cylinderepithelien mit ihren Flimmerbaaren, zum Theile im $\mathrm{Zu}$ stande schleimiger Entartung befindlich, bestehend fanden, während sich nur ganz wenig Eiterkörperchen nachweisen liessen. Jedenfalls ist hier die Oberfläche der Tube mit dem Messer abgestrichen und das Abgestrichene dann untersucht worden. 
weisse Blutzellen nicht sehr reichlich finden, ist das lockere Bindegewebe der Schleimhaut so dicht mit Rundzellen durchsetzt, dass von dem bindegewebigen Grundstocke nur stellenweise in der Achse der Zotten etwas zu sehen ist. Grössere Gefässe, erweiterte Capillaren sind nicht vorhanden.

Das Epithel ist an den tiefsten Stellen der Ausbuchtungen der Schleimhaut theilweise völlig nnversehrt; es bildet eine einfache Lage regelmässig geordneter Cylinderepithelien, an deren Rand ein hellerer Saum das Bestehen von Flimmerhaaren anzeigt. An den mittleren Theilen, besonders aber an den in die Höhlung hineinragenden Schleimbautzotten zeigt das Epithel eine ganz andere Beschaffenheit. Seine Zellen sind unregelmässig geordnet, vielfach durcheinander geworfen, an einzelnen Stellen durch lebhafteste Auswanderung weisser Blutzellen vollständig abgestreift. An den Stellen, wo noch Epithel vorhanden ist, sind die Zellen mehrfach geschichtet, von mehr cubischer Gestalt, von weissen Blutkörperchen reichlich durchsetzt und dadurch ausser Ordnung gebracht. Häufchen weisser Blutzellen (Eiter) liegen überall der Schleimhaut an. M a sse: Höhlung, nahezu kreisrund, $4 \frac{1}{2} \mathrm{~mm}$; Dicke der Ringmuskulatur 1-2 mm; Höhe der Schleimhantzotten $0,5-2,5 \mathrm{~mm}$; Höhe des Epithelbelages $30-60 \mu$.

2. Stïck aus derselben Tube, näher dem abdominalen Ende. Die Muskulatur erweist sich verdickt; ihre nach innen gelegenen Schichten sind stark infiltrirt, und zwar geht die Infiltration mit weissen Blatzellen unnittelbar über auf die Bindegewebszüge der Schleimhaut und ihrer Falten, wo sie so ausserordentlich dicht ist, dass vom eigentlichen Schleimhantgewebe nur an wenigen Stellen etwas zu sehen ist. Die Schleimhautfalten sind ungemein stark entwickelt, vielfach verästelt, und füllen die ganze Höhlung der Tube mit ihren papillösen Verästelungen nahezu vollständig aus. Besondere Gefässentwickelung tritt in der Schleimhaut nicht hervor. Die Epithelauskleidung der Schleimhaut ist in der Tiefe der Ausbuehtungen meistens normal, fehlt dagegen an der Spitze bezw. an den der Mitte der 'T'ubenhöllung zugekehrten Theilen der Schleimhautfalten fast vollständig. Es liegt dann das submucöse Bindegewebe, mit Eiterzellen vielfach durchsetzt, bloss. An anderen Stellen ist das Epithel durch die auswandernden Eiterkörperchen theils abgehoben, theils durcheinander geworfen: an wieder anderen Stellen findet sich ein polymorphes Epithel, bestehend theils ans mehr rundlichen, theils mehr abgeplatteten Zellen, die in unregelmässiger Schichtung übereinander gelagert sind, Allerorts liegen der Schleimhaut oberflächliche Eiterhäufehen auf. Maasse: Durchmesser der ovalen Höhlung 5-9 mm, Dicke der Muskulatur 1-2 mm, Zottenhöhe 2-5 mm, Epithelhöhe 26,5-32 $\mu$.

3. Stück aus der bei der Section herausgenommenen rechten Tube (etwa der Mitte entsprechend). Die Muskulatur erscheint ziemlich stark hypertrophiseh und ist von vielen und weiten Gefässen durchzogen. Auch in dem subserösen Bindegewebe findet sich eine auffallend starke Gefässentwickelung. Die allerinnersten Schichten der 
Muscularis sind infiltrirt; ausserordentlich starke Infiltration zeigt die Schleimhaut selbst. Thre Papillen sind nicht übermässig hoch, oft wie gedrückt und zeigen keine sehr starken baumartigen Verästelungen.

Das Cylinderepithel ist in der Tiefe der Ausbuchtungen meist normal, an der Oberfläche der Wülste aber vielfach abgestossen, so dass die infiltrirte Bindegewebslage blossliegt; oder es ist ein niedriges, polymorphes Epithel vorhanden, aus unregelmässig geformten, mehr rundlichen Bestandtheilen zusammengesetzt, zwischen dem viele Eiterzellen liegen. Maasse: Durchmesser der Höhlung 5-6 mm, Dicke der Muskulatur 1-21/2 mm, Zottenfläche 1-4 mm, Epithelhöhe 30 bis $38 \mu$.

Die Ergebnisse der Untersuchungen entsprachen den ursprünglich gehegten Erwartungen nicht. Es ist ja nicht wohl anzunehmen, dass der Verlauf der gonorrhoischen Infection in der Tube ein verschiedener sei von dem auf anderen Schleimhäuten; allein wir haben es hier jedenfalls schon mit dem Stadium der' Abheilung zu thun, und darin liegt wohl der Grund, warum 'sich nicht dieselben Veränderungen fanden, wie sie der acute Tripper auf der Schleimhaut hervorruft. Der Befund war denn auch, entsprechend dem Stadium der Krankheit, bei allen Präparaten ein verhältnissmässig gleichartiger.

Für alle drei Präparate ergiebt sich gemeinsam eine ziemlich gleichmässige, durch die das subseröse Bindegewebe, vor allem aber die Muskulatur betreffende Hypertrophie verursachte Verdickung der Tubenwandungen, die jedoch durchaus nicht äbermässig stark ist. Infiltration der Muskulatur mit Rundzellen ist ebenfalls allen Präparaten gemeinsam, sie erscheint nur im Präparat 3 als eine ausserordentlich starke, ist bei den übrigen eine mässige zu nennen und betrifft theilweise nur die innersten Schichten der Ringmuskulatur, theils die Spalträume zwischen den einzelnen Muskelzügen.

Die Gestaltung der Schleimhautwülste ist eine verschiedene. Von einer ausserordentlich starken Entwickelung sind dieselben in Präparat 2, wo sie die ganze Höhlung der Tube nahezu vollständig ausfüllen und lebhaft verästelt sind. Recht niedrig erscheinen sie dagegen in Präparat 1 und 3 ; sie sehen hier oft wie zusammengedrückt aus, sind nur spärlich verästelt; die Verästelungen selbst erscheinen oft kolbig verdickt. Von nahezu normaler Ausbreitung und Gestaltung scheinen sie nur in Präparat 1 zu sein.

Auffallend in allen Präparaten, besonders in Nr. 2, ist die ungemein starke Durchsetzung der Schleimhaut mit Rundzellen, die an vielen Stellen so ausserordentlich dicht ist, dass die bindegewebige Schicht der basalen Schleimhaut, wie vor allem der Zotten fast vollständig überdeckt erscheint. Zahlreich sind die Eiterhäufchen, die sich überall zwischen den einzelnen dendritischen Verzweigungen finden. 
Von der Mächtigkeit des Andranges der weissen Blutkörperchen giebt das Verhalten des Epithels in allen Präparaten gleichmässig Kenntniss: Auf den Erhebungen der Zotten wie auch anderen mittleren Theilen finden sich zahlreiche Stellen, die völlig entblösst sind von ihrem Epithel, so dass das bindegewebige Gerüst frei zu Tage tritt. In grossen Schollen ist es von den andrängenden Blutzellen abgehoben; wo das ursprüngliche Fipithel noch vorhanden ist, erscheint seine regelmässige Anordnung auf das empfindlichste gestört; die einzelnen Zellen sind aus der Reihe geworfen und liegen ordnungslos durch- und übereinander; die Flimmerhaare sind vielfach verschwunden. Dort aber, wo der Vorgang in Rückbildung begriffen ist, wo die Heilung beginnt, da findet sich das junge, weu gebildete Epithel in mehreren Lagen übereinander geschichtet, von den verschiedensten Formen; rundliche, abgeplattete und cubische Zellen sind an allen diesen Orten zu sehen, ohne Ordnung übereinander gelagert, um an der Grenze gegen die gesunden Stellen hin allmälig wieder in das typische, einschichtige, mit Flimmerhaaren versehene Cylinderepithel überzugehen. Dieser regelmässige Ban der Epithelschicht findet sich fast stets in den tieferen Buchten zwischen den einzelnen Wülsten, wo sie, trotz dichtester Infiltration des darunter gelegenen Bindegewebes, meist völlig unversehrt und unberïhrt von dem in den mehr peripher gelegenen Theilen so stürmisch verlaufenen Vorgange erscheint, Völlig gesundes Cylinderepithel, an dem meist auch noch die Flimmerhaare deutlich sind, findet sich auch in den offenbar durch die Verwachsung zweier oder mehrerer Schleimhautfalten entstandenen Hohlräumen.

Diese Hohlräume, welche verschiedene Forscher, Hennig z. B., an das Vorhandensein von Drüsen im Gewebe der Tubenwandungen glauben liessen, finden sich vorzugsweise an den Stellen, wo Falten von der wandständigen Schleimhaut sich erheben, aber auch in der Schicht der Fasern selbst, ja auch zwischen den Platten der Ringmuskulatur, in verschiedener Anzahl und Grösse; sie sind entstanden zu denken durch die infolge des vermehrten Inhaltsdruckes verursachte Verschmelzung einzelner Falten oder deren papillösen Verzweigungen untereinander.

Die auf dem Querschnitte rund, länglich oder oval erscheinenden Hohlräume sind als längsverlaufende, parallel der Höhlung sich hinziehende, von derselben völlig abgeschlossene Räume anzusehen. Diese Längsräume können dann ihrerseits wieder, wie es scheint, durch secundäre Wucherungen und Verschmelaungen in kleinere und kleinste Hohlräume zerlegt werden. Der Gedanke, dass man es hier wirklich mit Drüsen zu thun haben könne, wird widerlegt durch den völlig mit der Tubenschleimhaut übereinstimmenden Bau des Epithelüberzuges dieser Hohlräume, durch 
ihre oft recht beträchtliche Anzahl und Ausdehnung, die besonders in Präparat 3 eine sehr bedeutende war. ${ }^{1}$ )

Im wesentlichen stimmt also das Bild, welches die Untersuchung der gonorrhoisch erkrankten Tubenschleimhäute ergab, mit dem überein, welches das Verhalten des Gewebes, vor allem der Zustand des Epithels bei anderen gonorrhoisch inficirten Schleimhäuten bietet, bei denen der Vorgang sich in demselben schon weit vorgeschrittenen Stadium befindet, wie hier, und der Abheilung, der Wiedererneuerung sich nähert. Es ist deshalb auch anzunehmen, dass der Verlauf der frischen, acuten Gonorrhoe auf der Tubenschleimhaut ein gleicher ist, wie der auf den übrigen, gleich gebauten und der Infection zugänglichen Schleimhäuten.

Ein wichtiger Unterschied besteht jedoch: Während alle erkrankten Schleimhautstellen dem durch die massenhafte Auswanderung weisser Blutkörperchen in beträchtlicher Menge erzeugten Eiter unbehinderten Abfluss gestatten, ist der Eiterabfluss aus den Tuben in nicht geringem Grade erschwert. Auf der einen Seite das infolge entzündlicher Schwellung stark verengte ohnehin ja schon enge - Ostium uterinum; auf der anderen Seite das sehr oft verschlossen gewordene Ostium abdominale dadurch sind die Bedingungen im vollsten Maasse gegeben, den Eiterabfluss zu verhindern und die Bildung von Pyosalpinx herbeizuführen. Diese Entwickelung von Eitersäcken kann sowohl durch unmittelbare Verlöthung der Tubenwandungen unter sich, als auch durch Verwachsung des Ostium abdominale mit anderen Beckenorganen, z. B. den Ovarien, veranlasst sein.

Wie sich aus Salpingitis catarrhalis eine Hydrosalpinx, aus Salpingitis haemorrhagica eine Haematosalpinx gar nicht selten entwickelt, so ist auch die Pyosalpinx ein recht häufiger Ausgang der Salpingitis purulenta. Der durch die Zurückhaltung der Absonderung bei Pyosalpinx bedingte, langsam wachsende Inhaltsdruck verursacht durch die Zusammendrückung der Schleimhaut Schädigungen und Veränderungen derselben, die bei anderen Schleimhäuten natürlich fehlen, aber bei der Betrachtung des anatomischen Bildes wohl zu beachten sind.

1) Auch Frommel (Gynäkologische Gesellschaft zu München 1886) stellt das Vorhandensein von wirklichen Drüsen in Abrede, wie auch Chiari (Zeitschrift für Heilkunde, Bd. VIII) auf die mögliche Verwechselung dieser Ausbuchtungen und Abschnürungen in der entzündeten Tubenschleimhaut mit Drüsen aufmerksam macht. 
Ist der Verschluss des Ostium abdominale ein nicht ganz vollkommener oder nicht allzufester, so kann, besonders wenn irgend ein heftigerer Reiz einwirkt (Stoss oder Schlag, Ausschweifungen, Tanzen u. s. w.), eine gewisse Menge des im Tubensacke aufgestauten Eiters überfliessen, der in die Bauchhöhle gelangt und hier die bekannten schweren Complicationen der Gonorrhoe, auf welche ich noch zurückkommen werde, hervorruft.

Der Hauptzweck der angestellten Untersuchungen war, wie schon erwähnt, den bis jetzt noch nicht geglückten Versuch zu machen, die Gonococcen im Gewebe der Tuben - in deren Absonderung sie ja nachgewiesen waren -- aufzufinden. Ich will von vornherein anführen, dass es mir nicht gelungen ist, dieselben mit Sicherheit nachzuweisen.

Zur Färbung der Präparate bediente ich mich, auf den freundlichen Rath des Herrn Dr. Bumm hin, in ganz vorwiegender Weise der von ihm bei seinen Untersuchungen angewandten und fast einzig brauchbar gefundenen Methode. Die Präparate werden mit starken Lösungen von Methylenviolett in Toluidin- (oder Anilin-) Wasser gefärbt, welche man sich am zweckmässigsten vor dem Gebrauche jedesmal frisch bereitet, indem man von einer concentrirten alkoholischen Methylviolettlösung einige Tropfen in ein Schälchen mit vorher gekochtem und filtrirtem Toluidinwasser giebt.

Die Schnitte werden dann etwa eine halbe Stunde in der Färbeflüssigkeit belassen (erwärmt man die Farbstofflösung langsam und vorsichtig etwas, kann die Zeit abgekürzt werden). Zum Zwecke der Entfärbung kommen dann die Präparate in absoluten Alkohol, und hierin liegt die Schwierigkeit: den Zeitpunkt richtig zu exfassen, wo Kerne und Protoplasma schon ziemlich entfärbt, die Coccen aber, welche ihren Farbstoff nur ein wenig fester halten, noch gut gefärbt sind. Wird die Entfärbung zu früh abgebrochen, so ist an ein Erkennen der Coccen zwischen den noch dunkeln Kernmassen und dem noch gefärbten Protoplasma nicht zu denken; entfärbt man nur ein bischen zu lange, so sind auch die Gonococcen mit entfärbt und zu blass geworden, um sie erkennen zu können. - Nach der Färbung werden die Schnitte in Nelkenöl aufgehellt and in Canadabalsam eingeschlossen.

Nach dieser Methode nun wurden zahlreiche Tubenschnitte sorgfältig gefärbt und aufmerksam durchmustert; zur Vergrösserung 
bediente ich mich eines Seibert'schen Mikroskopes, homogene Immersion 1/12, nebst A b bé'schem Beleuchtungsapparate.

Da es mir nicht gelungen ist, auch nur in einem einzigen Schnittpräparate die Gonococcen zu finden, so muss ich annehmen, dass sie - bei dem ja schon ziemlich weit vorgeschrittenen Heilungszustande, in welchem die Tubenschleimhaut sich befand - wirklich nicht mehr im Gewebe der Tuben vorhanden, sondern zu Grunde gegangen waren, während sie sich im freien Secrete, wenn auch in nicht allzugrosser Zahl, noch erhalten hatten.

In den Schnittpräparaten können unter Umständen die sogenannten Ehrlich'schen Mastzellen das Vorhandensein von gonococcenhaltigen Zellen vortäuschen; die Aehnlichkeit ist oft eine recht grosse.

An die vorausgegangenen Erörterungen schliesse ich einige klinische Bemerkungen an über die Tubengonorrhoe, insbesondere über ihre häufigste Begleiterscheinung, die Perimetritis.

Ueber die Häufigkeit des Vorkommens der gonorrhoischen Erkrankung der Tuben gegenüber der des übrigen Genitalschlauches konnte ich weder aus der Literatur, noch aus den Journalen der Abtheilung für Syphilis des Juliusspitales, die mir von Herrn Professor Leube gütigst zur Verfügung gestellt wurden, sicher e Anhaltspunkte gewinnen. Der Grund liegt darin, dass in den meisten Fällen die ganz bestimmte Diagnose einer Erkrankung, speciell der Tuben, nicht gestellt wurde, sondern mehr im allgegemeinen die Erkrankung der Uterusanhänge und Umgebung. Die specielle Diagnose der Erkrankung der Tuben ist auch ausserordentlich schwierig, selbst unmöglich, so lange eine einfache Entzündung der Tubenschleimhaut und nicht schon die Bildung von Tubensäcken vorliegt.

Es standen mir im Ganzen 116 Fälle von Gonorrhoe beim Weibe zu Gebote. Bei 27 derselben wiesen die Erscheinungen auf eine secundäre entzündliche Erkrankung der Beckenorgane hin. Ausdrücklich hebe ich hervor, dass ich nur solche Fälle verwerthet habe, bei denen Gronococcen in der Absonderung der verschiedenen erkrankten Schleimhauttheile mit aller Sicherheit nachgewiesen wurden, ferner solche, bei denen nicht irgend eine andere Complication, welche Fieber, Entzündungserscheinungen u.s.w. erregen konnte, gleichzeitig bestand - mit Ausnahme von 4 gesondert anzuführenden Fällen von Syphilis -, und dass es sich end- 
lich in der Mehrzahl der Fälle um frische Infection, um acute oder wenigstens subacute Gonorrhoe handelte.

Es waren die folgenden:

1. 18 jährige Dienstmagd. Gonorrhoe der Urethra und der Cervix, reichliche Absonderung, viele Gonococcen. 14 Tage nach der Infection heftiges Erbrechen, starke Schmerzen im linken Parametrium. Im hinteren Scheidengewölbe starker Widerstand und Druckempfindlichkeit; Schmerzen in der linken Hüfte; diffuse Schmerzen im Unterleibe. Kurze schmerzfreie Zwischenzeiten. Temperatur: $38,3-39,8^{\circ}$; Puls bis 160. Nach zehn Tagen Besserung, geringes Fieber; Druckempfindlichkeit dauert fort. -

2. 20 jährige Dienstmagd. Gonorrhoe der Urethra und der Cervix; viele Gonococcen. Acht Tage nach dem letzten Beischlafe heftige Unterleibsschmerzen, besonders rechts. Innere Untersuchung, sowie Druck auf die Bauchdecken sehr schmerzhaft. Bei jeder Menstruation Vermehrung der Schmerzen. Uterus durch feste Verwachsungen hinten angeheftet; rechts von der Vaginalportion am Scheidengewölbe wallnussgrosse Cyste mit blutig schleimigem Inhalte. Temperatur 38,5 . -

3. 20 jährige Lustdirne. Massenhafte Gonococcen in der Absonderung der Urethra und Cervix. Drei Wochen nach der Infection beiderseits im Parametrium heftige Schmerzen, die drei Wochen fast ständig andauern, zeitweise schwinden, um bald wiederzukehren. Dauernd Temperatur 39,5. Der anfangs frei bewegliche Uterus wird bald befestigt, ist sehr schmerzhaft. Im Ligamentum latum sinistrum hühnereigrosser 'Tumor; Ligamentum latum dextrum verdickt. Nach acht Tagen ist der Tumor verschwunden, vier Wochen später das vordere Scheidengewölbe mit zwei hühnereigrossen Exsudaten gefüllt. Rechts ein deutlich abgrenzbarer Strang. Schmerzhaftigkeit und Fieber wechseln mit nahezu freien Zeiten ab. -

4. 16 jährige Arbeiterin. Viele Cocen in der Absonderung der Cervix. Sechs Wochen nach der Infection starke Schmerzen im rechten Perimetrium. Das rechte Scheidengewölbe steht viel tiefer als das linke, ist widerstehend und druckempfindlich. 14 Tage lang bestehen links auch Schmerzen. Temperatur 38,7. -

5. 28 jährige Näherin. Gonorrhoea cervicis. Condylomata acuminata, die abgetragen und geätzt werden. Etwa vier Wochen nach der Infection sehr schlechtes Allgemeinbefinden, tiefe Blässe im Gesichte, unerträglicher Kopfschmerz, Schwindel. Heftige Schmerzen im Unterleibe, Erbrechen, Athemnoth. (Keine innerliche Untersuchung wegen übergrosser Schmerzhaftigkeit.) Trismus, Tod. Section: Der Ueberzug der linken Tube geröthet, die Tube geschwellt, geschlängelt verdickt. Rechte Tube äusserlich weniger ergriffen. Die Schleimhaut beider Tuben fleckig tief geröthet; beim Aufschneiden quillt reichlich trübe, eitrige Flüssigkeit heraus; keine besondere Exsudatbildung. - 
6. 16 jährige Dienstmagd. Gonorrhoea cervicis mit vielen Gonococcen. Vier Wochen nach der Infection heftige Schmerzen im Unterleibe; Perimetrium sehr schmerzhaft und widerstehend; reichlicher Durchfall; mässiges Fieber. -

7. 17 jährige Dienstmagd. Im Urethral- und Cervicalsecrete massenhafte Gonococeen. Sechs Wochen nach der Infection ausserordentlich schlechtes Allgemeinbefinden, besonders im Anschlusse an die Regel, grosse Mattigkeit, unruhiger Schlaf, Appetitmangel; starke Schmerzhaftigkeit in den Parametrien, die eine genaue innerliche Untersuchung unmöglich macht. Temperatur $38,8-39,5$. -

8. 20 jährige Dienstmagd. Zweite gonorrhoische Infection; Zeit nicht nachweisbar. Die Absonderung der Urethra hat reichlich Gonococcen. Starke Schmerzen im Parametrium, besonders links erhebliche Verhärtung ohne besondere Hervorragung; Stuhl bald dnrchfällig, bald stark verzögert. Temperatur $38,5-39,6$. -

9. 29 jährige Taglöhnerin. In der Absonderung der Urethra Gonococcen. Sehr schlechtes Allgemeinbefinden, Appetitmangel, Schlaflosigkeit. Heftige Schmerzen im ganzen widerstehenden Parametrium. Dauernd mässige Temperatursteigerungen. -

10. 19 jährige Dienstmagd. In der reichlichen Absonderung der Urethra and Cervix viele Gonococeen. Zeit der Infection nicht nachweisbar. Aeusserst schlechtes Allgemeinbefinden; Parametrium sehr schmerzhaft und widerstehend; der Absonderung der Cervix ist öfter Blut beigemischt. Temperatur stets 39,5 . -

11. 19jährige Dienstmagd. Absonderung der Urethra und Cervix führt viele Gonococcen. 14 Tage nach der Infection Durchfälle, Appetitlosigkeit, grosse Mattigkeit; heftigste Schmerzen im kleinen Becken. Temperatur bis 39,1 . -

12. 19jährige Näherin. Gonorrhoe der Urethra. Zwei Monate nach dem letzten Beischlafe heftige Schmerzen im Unterleibe bezw. Parametrium. Douglas'scher Raum, besonders rechts, sehr schmerzhaft. Uterus stark nach hinten gebeugt, angeheftet, sehr verhärtet und schmerzhaft. Temperatur erhöht: 38,2 ; sehr oft bis 40,0 steigend. -

13. 19 jährige Dienstmagd. Urethritis et Inflammatio glandulae Bartholini gonorrhoica mit vielen Gonococcen. Zwei Monate nach der Infection sehr heftige Schmerzen im rechten Parametrium. Milz leicht vergrössert; heftiger Kopfsehmerz. Mit jeder Regel werden die Schmerzen im Parametrium fast unerträglich. Temperatur lange Zeit hindurch $38,0-39,0$.

14t. 23jährige Dienstmagd. Gonorrhoea cervicis. Etwa 14 Tage nach der Infection starke Schmerzen im Unterleibe. Tastung des Bauches, besonders in den unteren Theilen, sehr schmerzhaft. Percussion ergiebt beiderseits, besonders links unten Dämpfung. Starke Schmerzen im Parametrium. Uterus nach hinten gebeugt, unten frei, oben angeheftet; bei Berïhrung höchst schmerzhaft. Die Schmerzen 
im Unterleibe wechseln an Heftigkeit, mit jeder Schmerzsteigerung aber tritt Temperaturerhöhung bis 39,4 ein. -

15. 20jährige Kellnerin. Zweite gonorrhoische Infection, Zeit nicht zu bestimmen. Gonorrhoea cervicis, spärliche Gonococen. Heftige Schmerzen im Unterleibe, Erbrechen; Uterus sehr stark nach hinten gebeugt, unbeweglich, sehr schmerzhaft. Do u gl a s'scher Ranm frei; im unteren Theile des linken Ligamentum latum mässig grosses Exsudat. Hohe Temperaturen. -

16. 19jührige Näherin. Gonorrhoea cervicis. Zeit unbestimmt. Ausserordentlich heftige Schmerzen beiderseits im Bauche; Parametrien sehr widerstehend und schmerzhaft. Temperatur lange Zeit 38,5-40,0.

17. 19 jährige Kranke. Gonorrhoea urethrae et cervicis; massenhafte Gonococcen; Infectionszeit nicht nachzuweisen. Leidet zeitweise an hysterischen Krampfanfällen, die, wie es scheint, erst seit der Infection auftraten. Starke Schmerzen in den unteren Theilen des Bauches. Portio vaginalis nach rechts verdrängt, Seheidengewölbe höchst schmerzhaft und steht links bedeutend tiefer als rechts. Temperatur bis $38,8^{\circ}$.

18. 18jähriges Dienstmädchen. Gonorrhoea urethrae mit sebr vielen Coccen. Sehr starke Schmerzen im Unterleibe, schlechtes Allgemeinbefinden. Temperatur $39,6^{\circ}$. - Uterus stark verhärtet; Parametrium sehr widerstehend and angemein druckempfindlich.

19. 18 jährige Magd. Gonorrhoe der Urethra und Cervix, viele Gonococcen. Drei Wochen nach der Infection sehr heftige Leibschmerzen, Bauch unten sehr druckempfindlich. Uterus stark nach vorn gebeugt, angeheftet und schmerzhaft. 14 Tage lang Abendtemperatur: $39,5-40,0$.

20. 23jährige Dienstmagd. Gonorrhoea cervicis. Acht Tage nach dem letzten Beischlafe (wahrscheinlich hierbei Infection): Schmerzen bei Druck auf den Bauch. Rechts und links unten percussorisch Dämpfung. Uterus spitzwinkelig nach hinten gebeugt; erheblich vergrössert. Portio vaginalis frei beweglich. Uterus im Douglas'schen Raume durch fühlbare straffe Verwachsungen angeheftet; sehr druckempfindlich. Temperatur: bis 39,8 .

21. 19jährige Dienstmagd. Gonorrhoea cervicis; wenig Gonococcen. Drei Monate nach der Infection treten heftige Schmerzen unten im Bauche auf, Allgemeinbefinden schlecht. Scheidengewölbe vorgewölbt, widerstehend. Temperatur: bis 39,9 .

22. 23jährige Lustdirne, hat ein gesundes Kind. Gonococeen massenhaft in der Absonderung der Urethra und Cervix. Zeit der Infection nicht nachweisbar. Beiderseits das Parametrium verdickt, widerstehend, sehr druckempfindlich. Berührung des leicht angehefteten Uterus ungemein schmerzhaft. Kopfweh, Erbrechen. - Temperatur: bis 39,4 .

23. 18jährige Dienstmagd. Gonorrhoea urethrae et cervicis, viele Gonococeen. Fünf Monate nach der Infection, während welcher Zeit der Tripper fortdauerte, heftige Schmerzen im Bauche. Uterus 
sehr schmerzhaft. Parametrien sehr widerstehend und druckempfindlich. Temperatur: bis 39,8 .

$\mathrm{Zu}$ diesen 23 Fällen von reiner Gonorrhoe ohne jede weitere Complication kommen noch 4, bei denen früher Syphilis bestanden hatte (2) oder noch gleichzeitig mit Gonorrhoe besteht (2).

Es bestand wohl in einer grösseren Anzahl der von mir durchgesehenen Fälle neben Syphilis auch Gonorrhoe; bei der weitaus überwiegenden Zahl aber traten die syphilitischen Erscheinungen so sehr in den Vordergrund, dass ich nur folgende 4 Fälle mit unter die Gesammtzahl (116) einrechnen konnte:

1. 18jährige Lustdirne. War vor zwei Jahren syphilitisch inficirt und wurde mittels Inunctionseur geheilt. Vor 1/2 Jahre Geburt eines gesunden, völlig ausgetragenen Knaben. Zur Zeit besteht Gonorrhoe der Cervix mit vielen Gonococen in der Absonderung. Wann Infection stattfand, jst nicht zu bestimmen. Es traten heftige Schmerzen im Unterleibe ein, die zeitweise verschwinden, dann aber bald mit erneuter Heftigkeit wiederkehren. Uterus stark vergrössert, sehr schmerzhaft. Rechtes Ligamentum latum infiltrirt, verdickt, verhärtet. Temperatur: bis 39,6 .

2. Kranke seit längerer Zeit syphilitisch. Im Anschlusse an eine Frühgeburt im sechsten Monate war ein mässig grosses Exsudat entstanden, das nach 14 Tagen völlig verschwunden gewesen sein soll. Die Zeit der gonorrhoischen Infection (Gonorrhoea cervicis mit vielen Gonococcen) ist nicht sicher zu bestimmen; kurze Zeit nach dem wahrscheinlich inficirenden Beischlafe entstand ein sehr grosses Exsudat, bei heftigen Schmerzen. Seitliche Stellung, Vergrösserung, Verhärtung und grosse Schmerzhaftigkeit des Uterus. Stark fieberhafte Temperaturen.

3. 20jährige Lustdirne. Hatte wiederholt Gonorrhoe und wird zur Zeit auch wegen Syphilis behandelt. Während der jetzt bestehenden Gonorrhoe (Gonorrhoea cervicis) traten sehr heftige Schmerzen im Unterleibe auf, Erbrechen. Der Uterus ist durch starke Verwachsungen nach rechts gezogen, fast ganz unbeweglich. Am unteren Theile des linken Ligamentum latum mässig grosses Exsudat; links besonders starke Schmerzen. Sehr hohes Fieber.

4. 20jährige Lustdirne wurde in ihrem 17. Jahre wegen Syphilis behandelt und geheilt. In der Absonderung der Urethra und Cervix viele Gonococeen. Starke lang dauernde Blutungen aus dem Uterus, der erheblich nach rechts verdrängt ist. Linkes Scheidengewölbe steht viel tiefer als das rechte und ist ungemein druckempfindlich. Bauch bei Berührung sehr schmerzhaft. Mässiges Fieber: bis 38,7.

In den 116 Fällen, bei denen die Diagnose „Gonorrhoe“ durch den unzweifelhaften Nachweis des specifischen Mikroorganismus als vollständig gesichert angesehen werden muss, traten also $23 \mathrm{Mal}$, 
und mit Einschluss der Fälle, bei denen Syphilis bestand oder bestanden hatte, $27 \mathrm{Mal}$ - im Ganzen in 19,8 Proc. bezw. in 23,3 Proc. der Gesammtzahl - Erscheinungen auf, die auf eine Weiterverbreitung der Gonorrhoe über den Uterus auf dessen Anhänge - im weitesten Sinne - hinweisen; Erscheinungen, die schwerwiegend genug sind, und eine Zahl, gross genug, um der vollen Beachtung werth zu sein.

In wie vielen Fällen wirklich eine Tubengonorrhoe vorhanden war, ist natürlich sehr schwer zu sagen. Bedenkt man jedoch, dass in vielen Fällen nach verhältnissmässig kurzer Zeit Heilung eintrat, dass ferner entzündliche Vorgänge im Parametrium und im Beckenperitoneum auch ohne Tubengonorrhoe sehr oft vorkommen, besonders bei Personen, die so vielen Eingriffen ausgesetzt sind, wie die Lustdirnen, so dürfte sich das Procentverhältniss, in welchem sich Tubengonorrhoe mit der Gonorrhoe des Genitaltractus überhaupt verbindet, sehr viel niedriger stellen als 19,8, bezw. 23,3 Proc., ja vielleicht über 5 Proc. kaum hinaufgehen.

Auf die Anschauungen der verschiedenen Forscher über die Häufigkeit, in welcher die Gonorrhoe beim Weibe auf den ganzen Genitalschlauch, im besonderen auf die Tuben und von da weiter sich fortsetzt, habe ich oben kurz hingewiesen. Abgesehen von der in ihrer Allgemeinheit gewiss zu weit gehenden und in ihrer Ausdehnung gar nirgends bestätigten Ansicht Nöggerath's, dass jede zu irgend einer Zeit einmal gonorrhoisch inficirte Frau ,mit voller Sicherheit" auf eine Weiterverbreitung der Erkrankung zu rechnen habe, dass also in allen, oder doch in fast allen Fällen die Gonorrhoe den Uterus, die Tuben und von da das Peritoneum befalle; abgesehen ferner von der Ansicht Sänger's, der sich an Nöggerath im wesentlichen anschloss, wenn er auch nur annimmt, dass in allen Fällen die Tuben und die übrigen Uterusadnexe ergriffen werden können, findet sich an keinem. Orte, so weit mir die Literatur zugänglich war, ein durch bestimmte Zahlen und durch das Auffinden des Gonococcus gesicherter Nachweis, wie oft denn in Wirklichkeit ein Ergriffenwerden der Tuben eigentlich statthat. Aber alle Forscher weisen ausdrücklich auf die grosse Häufigkeit dieser Verbindung hin und auf den grossen Procentsatz, mit dem die Gonorrhoe bei allen acut oder chronisch auftretenden Entzündungserscheinungen des Beckenperitoneum und der Tuben (nach Martin sind $5 / 12$ aller Fälle von Tubenerkrankung gonor- 
rhoischen Ursprunges, nach Sänger die weitaus meisten) betheiligt ist, sowie auf die hohe Wichtigkeit, welcher der Gonorrhoe als ätiologisches Moment bei allen derartigen Erkrankungen zukommt.

Einen Punkt möchte ich zur Beurtheilung der oben angeführten Zusammenstellung noch hervorheben. Es handelt sich in diesen Fällen um Kranke, welche in einer unzweifelhaft grossen Anzahl der öffentlichen oder geheimen Prostitution ergeben sind, so dass bei ihnen die von Vielen geforderten, zur Ausbreitung der Infection bis in die Tuben sicher begünstigenden Umstände, vor allem die Ausschweifungen in venere, Angriffe u. s. w., in reichlichem Maasse gegeben sein dürften.

Von einigem Interesse ist es vielleicht noch, die $\mathrm{Zeit} z u$ beachten, innerhalb welcher die entzündlichen Erscheinungen an den Uterusanbängen nach der gonorrhoischen Infection auftraten.

Eine' ganz genaue Zeitangabe ist natürlich ungemein schwer zu machen, wenn nicht unmöglich, da derartige Entzündungserscheinungen recht wohl schon einige Zeit bestanden haben können, ohne zur Beobachtung gelangt zu sein. Ob man aus der Zeit und Raschheit des Auftretens solcher Erscheinungen vom Anfange der Infection an Schlüsse auf die Heftigkeit derselben, oder auf bestimmte, die Weiterverbreitung befördernde Umstände zu ziehen berechtigt ist, muss ich dahingestellt sein lassen. Die ersten und vielleicht auch einzigen Autoren, welche ihr Augenmerk auf diesen Punkt; richteten, waren Bernutz und Goupil. ')

Waren die von ihnen beobachteten 28 Fälle von Peritonitis im Anschlusse an Blennorrhoe wirklich gonorrhoischer Natur bei dem natürlich mangelnden Nachweise des specifischen Mikroorganismus dürften Zweifel obwalten —, so trat diese Verbindung nach folgenden Zeiten ein:

\begin{tabular}{|c|c|c|c|c|c|c|c|c|}
\hline $\mathrm{Ann}$ & 8.-10. & Tage & vom & Anfange & der & Gonorrhoe & an in 1 & 1 Falle, \\
\hline g" & 12. & $"$ & $" 9$ & $"$ & $"$ & $"$ & $" \quad, 1$ & $1 \quad$ \\
\hline$"$ & 15. & $"$ & $"$ & $"$ & $"$ & $"$ & , & 3 Fällen, \\
\hline " & 21. & $"$ & $"$ & $"$ & " & $"$ & $"$ & 1 Falle, \\
\hline zu. & Ende des & s 1. Monates & $"$ & $"$ & $"$ & , & $"$ & 7 Fällen, \\
\hline$"$ & $\begin{array}{ll}\prime & \text { der } \\
. & \text { des }\end{array}$ & $\begin{array}{l}\text { r 6. Woche } \\
\text { s 2. Monates }\end{array}$ & ". & $"$ & $"$ & $"$ & ", & 1 Falle, \\
\hline$"$ & det & to a. Inullates & " & " & $"$ & $"$ & $"$ & \\
\hline
\end{tabular}

Bei den übrigen 13 Fällen war eine genauere Zeitbestimmung nicht möglich.

1) a. a. 0 . 
In meinen 27 Fällen konnte (einschliesslich der vier mit Syphilis verbundenen) im Ganzen $12 \mathrm{Mal}$ die Zeit der Infection aus verschiedenen Gründen nicht genau ergründet werden. In den übrigen 15 Fällen verhält sich die Zeit des Auftretens von Complicationen nach der Infection folgendermaassen:

In 8 Tagen vom Anfange der gonorrhoischen Infection an in 2 Fällen,

\begin{tabular}{|c|c|c|c|c|c|c|c|c|c|}
\hline, 14 & & $"$ & $"$ & $\because$ & $"$ & $"$ & $"$ & $n$ & 3 \\
\hline & Wochen & $"$ & $n$ & $n$ & $n$ & $"$ & יי & $n$ & 2 \\
\hline 4 & $"$ & " & $"$ & $n$ & $"$ & $"$ & ", & $"$ & 2 \\
\hline 6 & $"$ & , & $"$ & " & " & $"$ & " & " & 2 \\
\hline & Monaten & $n$ & "s & $"$ & $"$ & $"$ & $"$ & $"$ & 2 \\
\hline 3 & $"$ & $"$ & $"$ & $"$ & $"$ & $"$ & $"$ & $"$ & \\
\hline
\end{tabular}

Es zeigt sich also, dass die Weiterverbreitung der gonorrhoischen Infection über die ganze Schlèimhaut des weiblichen Genitalapparates und auf die benachbarten Beckenorgane durchaus nicht an eine bestimmte Zeit gebunden ist, wohl aber scheint dieses Fortschreiten in der Mehrzahl der Fälle innerhalb der ersten zwei Monate stattzufinden, wie aus den beiden oben angeführten Zusammenstellungen hervorgeht (nur in zwei von meinen Fällen trat es später ein); es ist auch von vornherein anzunehmen, dass das acut entzündliche Stadium der Erkrankung grössere Neigung zur Ausbreitung hat, als das chronische, bei welchem die Virulenz der Gonococcen doch wohl schon verringert ist.

Zum Schlusse noch einige Worte über die Entstehungsart der Perimetritis gonorrhoica, wie sie im Anschlusse an die gonorrhoische Tubenerkrankung so ungemein häufig auftritt. Für das Entstehen von Perimetritis gonorrhoica sollen nach"Fritsch u. A. hauptsächlich die Lymphbahnen den Weg abgeben, auf welchem die Gonococcen vordringend diese Entzündung erregen. Doch ist ein gleiches Verhalten dafür, dass der Mikroorganismus der Gonorrhoe von einer etwa in der Schleimhaut vorhandenen Wunde aus in die Lymphbahnen ein und in ihnen weiter vordringt, wie es z. B. bei Staphylococcus pyogenes aureus u. s. w. angenommen werden darf, für den Gonococcus bis jetzt nicht bekannt. Ausserdem aber ist die von Fritsch gezogene Parallele zwischen Perimetritis und den bei Tripper häufig auftretenden Entzündungen der Leistendrüsen insofern nicht stichhaltig, als in dem Eiter dieser sogenannten Tripperbubonen das Vorhandensein von Staphylococcus aureus und damit das Bestehen einer Mischinfection, auf welche diese Entzündungen zurückzuführen sind, mit aller Sicherheit nachgewiesen ist. 
Es bleiben also, wie mir scheint, nur noch zwei. Wege übrig, auf welchen das Entstehen einer Perimetritis im Anschlusse an Gonorrhoe möglich und erklärlich ist, und zwar 1) dass die Entzündung angeregt wird durch unmittelbare Fortleitung des virulenten Katarrhes durch die Tubenwand hindurch. Die Möglichkeit, dass die in der Tube bestehende Entzündung durch die verhältnissmëssig düme Tubenwandung hindurch auf das dicht anliegende Peritoneum sich fortsetzen und hier Reizerscheinungen, d. h. Entzündungen hervorrufen könne, ist ja gewiss nicht vou der Hand zu weisen; sicher aber ist, dass von einem Durchwandern der Gonococcen durch die Wand und von einer unmittelbaren Infection durch dieselben nicht die Rede sein kann, einfach deshalb, weil ihm, wie schon öfter betont, die Kraft fehlt, tiefere Bindegewebsschichten zu durchdringen und sich hier zu vermehren.

Es ist also noch 2) das unmittelbare Ueberfliessen des gonorrhoischen Eiters in die Bauchhöhle zu berücksichtigen, ein Ereigniss, das von fast allen Forschern, so besonders von Hennig, Nöggerath, Sänger, Veit, Gusserow u. A. als das hauptsächlichste Moment für das Entstehen einer gonorrhoischen Perimetritis dargestellt wird. Es ist mit grosser Sicherheit anzunehmen, dass besondere Bedingungen dieses Ueberfliessen des Eiters herbeizuführen im Stande sind, so vor allem Ausschweifungen und Angriffe aller Art, starke Gemüthsaufregungen, heftiger Schreck (Nöggerath führt einen derartigen sehr deutlich sprechenden Fall æn), Tanz, schwere Arbeit, übermässiger Alkoholgenuss.

Das unter allen diesen Bedingungen wiederholt erfolgende Ueberfliessen des Eiters, z. B. aus einer durch die zuerst hervorgerufene perimetrische Entzündung veranlassten Pyosalpinx, ist jedenfalls auch das Hauptmoment bei der Entstehung der rückfälligen Perimetritis.

Es ist zwar auch bei diesem Vorkommniss eine unmittelbare Einwirkung des Gonococcus im allerhöchsten Grade unwahrscheinlich. Denn das Plattenepithel, das dem Bauchfelle eigen ist, ist ein ebenso sicherer Schutz gegen das Eindringen dieses Mikroorganismus, wie z. B. das der Vagina.

So wenig man bei den infolge der ätzenden Wirkung des Trippereiters an den äusseren Genitalien entstandenen Entzündungserscheinungen an eine unmittelbare Gonococcenwirkung denken kann, ebensowenig ist an eine eigentlich gonorrhoische, unmittelbar durch Gonococcen erzeugte Perimetritis zu denken. Es ist daher 
anzunehmen, dass auch die nach Ueberfliessen gonorrhoischen Tubensecretes in die Peritonealhöhle entstandene Perimetritis nicht als eine gewissermaassen durch Impfung hervorgerufene specifische Erkrankung anzusehen ist, dass es sich vielmehr um eine durch den Reiz des virulenten Eiters erzeugte einfache Entziindung handelt, die sich durch Exsudatbildung, Verwachsungen u. s. w. äussert.

Das Vorhandensein von Gonococcen in diesem Eiter ist also ohne jeglichen Einfluss auf das Entstehen von Perimetritis; ob der etwa von den Gonococeen erzeugten Ptomaine eine derartige Wirkung zugemessen werden darf, ist wegen des bis jetzt völlig mangelnden Bekanntseins derselben nicht zu entscheiden. Man könnte sich vielmehr die Wirkung des übergeflossenen Eiters etwa so vorstellen, wie die Wirkung einer auf das Peritoneum gebrachten starken Säure oder irgend eines anderen reizend wirkenden aseptischen Fremdkörpers: Hier wie dort dieselben Erscheinungen, Entzündung, Exsudatbildung, Abkapselung des Exsudates u. s. w. Ein Beweis dafür, dass das Peritoneum gewissermaassen geschützt ist gegen die eitrige gonorrhoische Absonderung der Tuben. Der Beweis für die nicht infectiöse Natur dieses Eiters wird übrigens auch durch die Thatsache geliefert, dass z. B. bei Salpingotomien oft genug der Inhalt geplatzter Tubensäcke in die freie Bauchhöhle sich ergiesst, ein Vorkommniss, auf dessen verhältnissmässige Unschädlichkeit Gusser ow ${ }^{1}$ ) ausdrücklich hinweist.

Keinesfalls ist allerdings zu bezweifeln, dass eine grosse Zahl von Frauen, die an chronischen Perimetritiden litten, oft ganz plötzlich unter den Erscheinungen heftigster septischer Peritonitis zu Grunde gehen, als deren Ursache bei der Section sich das Platzen einer Pyosalpinx ergiebt, das oft ohne jeden nachweisbaren Grund, manchmal im Anschlusse an höchst unbegründete Operationen erfolgt ist. Bezüglich des letzteren Umstandes ist aber in vielen Fällen das Eindringen anderer Milkroorganismen, also eine Mischinfection, recht wohl als mögliche Ursache anzusehen.

1) Dieses Archiv 1887 und 1888, Bd. XXXII. 